\title{
Diagnose et écologie de quelques microalgues de la lagune Vodroboué et son embouchure
}

\author{
${ }^{1 *}$ ADON Marie Paulette, ${ }^{2}$ SALLA Moreto, ${ }^{3}$ KONAN Estelle Sévérine, ${ }^{4}$ KOMOÉ Koffi, \\ ${ }^{5}$ OUATTARA Allassane, ${ }^{5}$ GOURENE Germain \\ 1UFR - Environnement, Université Jean Lorougnon Guédé, BP 150 Daloa, Côte d'Ivoire. Département: Biodiversité et \\ Gestion Durable des Ecosystèmes. Enseignante-Chercheur attaché au Laboratoire d'Environnement et de Biologie Aquatique, \\ Université Nangui Abrogoua, Abidjan, Côte d'Ivoire, \\ 2 UFR - Agroforesterie, Université Jean Lorougnon Guédé, BP 150 Daloa, Côte d'Ivoire. \\ 3 Centre de Recherches Océanologiques, 29, Rue des Pêcheurs Treichville BPV 18 Abidjan, Côte d'Ivoire. \\ 4 Laboratoire de botanique, Université Félix Houphouët Boigny, 22 B.P. 582 Abidjan 22, Côte d'Ivoire. \\ 5 Université Nangui Abrogoua, UFR Science et Gestion de l'Environnement, Pôle Pêche et Aquaculture/Laboratoire \\ d'Environnement et de Biologie Aquatique, 02 BP 801 Abidjan 02, Côte d'Ivoire. \\ Auteur correspondant email : adonmariepaulette@gmail.com,Tel: (225) 075719 13; Fax: (225) 32787570
}

Mots-clés : Phytoplancton, épiphyton, physico-chimie, lagune, Côte d'Ivoire.

Keywords: Phytoplankton, epiphyton, physico-chimical, lagoon, Côte d'Ivoire.

Publication date 30/11/2019, http://www.m.elewa.org/JAPS

\section{RESUME}

La description succincte et l'écologie d'un taxon de Dinophyta et de quelques taxons de Chrysophyta ont été réalisées à partir du microscope électronique à balayage (MEB) et des paramètres physico-chimiques. Les mesures des paramètres physico-chimiques (Température, $\mathrm{pH}$, conductivité, nitrates et phosphates) et l'échantillonnage des taxons ont été réalisés entre $8 \mathrm{~h}$ et $12 \mathrm{~h}$ dans trois stations de la lagune Vodroboué (V1, V2 et V3) et son embouchure (E) pendant les périodes 2014-2015 et 2016-2017. Les échantillons pour le phytoplancton ont été prélevés à l'aide de la bouteille hydrologique, puis filtrés avec le filet à plancton. L'épiphyton a été récolté sur des macrophytes localisés dans le plan d'eau lagunaire à l'embouchure et au niveau des berges des stations V1, V2 et V3. Les taxons Torodinium sp. de la classe des Dinophyceae, Paraphysomonas uniformis hemiradia sp. $\mathrm{n}$. Type de la classe des Chrysophyceae, les écailles de Mallomonas sorohexareticulata Jo, Shin, Kim, Siver \& Andersen sp. nov., Mallomonas sp. et Mallomonas sp. 1 de la classe des Synurophyceae ont été observés pour la première fois en Côte d'Ivoire. Selon la diagnose des taxons, une similarité de l'ultrastructure des écailles de Mallomonas sorohexareticulata sp. nov. d'une part, et d'autre part, celles de Mallomonas sp. serait liée à la tolérance à la température. Pour ce qui concerne leur écologie, les taxons ont tolérés des pH acidoalcalines, une faible conductivité, une température et des taux de nutriments modérés. Les résultats de cette étude contribuent à la connaissance de quelques taxons de Dinophyta et de Chrysophyta qui pourraient être utilisés pour l'enseignement et les études de taxonomie des microalgues. 
Diagnosis and ecology of some microalgae from the Vodroboué lagoon and around its mouth

\begin{abstract}
Succinct description and ecology of one Dinophyta taxa and some Chrysophyta taxa were realized from scanning electron microscope (SEM) and physico chemical parameters. Measurements of physicochemical parameters (temperature, $\mathrm{pH}$, conductivity, nitrates and phosphates) and sampling of taxa were carried out between 8 and 12 in three stations of the Vodroboué lagoon (V1, V2 and V3) and its mouth (E) during 2014-2015 and 2016-2017 periods. Samples for phytoplankton were collected using the hydrologic bottle and then filtered with the plankton net. The epiphyton was collected by expression method on macrophytes located in the lagoon at the mouth and at the banks of V1, V2 and V3. Torodinium sp. of the Dinophyceae class, Paraphysomonas uniformis hemiradia sp. n. Type of Chrysophyceae class, the scales of Mallomonas sorohexareticulata Jo, Shin, Kim, Siver \& Andersen sp. nov., Mallomonas sp. and Mallomonas sp. 1 of the class Synurophyceae were observed for the first time in Ivory Coast. According to the diagnosis of taxa, a similarity of the ultrastructure of Mallomonas sorohexareticulata sp. nov. scales, on the one hand, and Mallomonas sp. scales on the other hand would be related to temperature tolerance. Concerning their ecology, taxa have tolerated acido-alkaline $\mathrm{pH}$, low conductivity and moderate temperature and nutrient levels. Results of this study contribute to the knowledge of some Dinophyta and Chrysophyta taxa can be used for teaching and microalgae taxonomy studies.
\end{abstract}

\section{INTRODUCTION}

Les environnements lagunaires, de par leur nature fertiles, fournissent des abris et des aliments pour de nombreux organismes aquatiques favorisant la mise en place dans de nombreux pays de grandes industries de pêche (Laë, 1992). Parmi ces aliments, les microalgues, organismes unicellulaires photosynthétiques constituent la base de la chaîne alimentaire en milieu aquatique. Leur classification est complexe faisant appel à des critères morphologiques et métaboliques (Person, 2011). En Côte d'Ivoire, peu d'études systématiques menées sur les microalgues ont portées sur la technique d'utilisation du microscope électronique à balayage (M.E.B.) (Da, 2007 ; Lozo et al., 2013) mettant en exergue l'ultrastructure des taxons observés. Ce M.E.B. (Philibert, 1968) est une technique de microscopie électronique capable de produire des images en haute résolution de la surface d'un échantillon en utilisant le principe des interactions électrons-matière. Ce microscope a pour avantage par rapport au microscope optique le grossissement continûment variable d'environ 100 à 30000 fois ou plus et une importante profondeur de champ à l'opposé du microscope photonique (M.P.). En outre, ce M.E.B. constitue une méthode d'examen non destructif dans une très large gamme de grandissements. Cet appareil peut donc être utilisé comme un complément du microscope métallographique, surtout $\mathrm{du}$ fait de ses possibilités de grandissement. Au niveau de la lagune Vodroboué dont plus de 400 taxa ont été récoltés regroupant le phytoplancton et l'épiphyton, la nécessité d'utiliser le M.E.B. était de mettre en exergue l'ultrastructure de quelques taxons nouvellement rencontrés dans la lagune et son embouchure. Les microalgues planctoniques ont non seulement une grande importance dans le fonctionnement des écosystèmes aquatiques, mais aussi un grand potentiel pour la production de composés intéressants pour l'alimentation humaine et l'aquaculture (Röch et Posten, 2012), et pour la production de molécules à haute valeur ajoutée 
dans l'industrie pharmaceutique et cosmétique (Spolaore et al, 2006 ; Brennan et Owende, 2010). En revanche, les microalgues peuvent présenter des risques de toxicité (efflorescences ou bloom) préjudiciables aux autres organismes vivants (Guallar-Morillo et al., 2015), tandis que les proliférations de microalgues nuisibles sont considérées comme un des principaux risques

\section{MATERIEL ET METHODES}

3.1 Situation géographique de la zone d'étude et choix des stations de prélèvement : La lagune Vodroboué (Fig. 1), de coordonnées 435000 UTM latitude Est et 579000 UTM longitude Nord, est localisée dans la zone humide estuarienne de Grand-Bassam, localisée dans le département de Grand-Bassam de coordonnées UTM (WGS84): Zone 30N E: 416479,74 N: 577689,04, inscrite à la convention de Ramsar (FDR, 2005). Cette lagune, couvrant une superficie de 423 ha avec une profondeur moyenne de $4 \mathrm{~m}$, est caractérisée par 2 saisons pluvieuses (avril-juin et octobre-novembre) et 2 saisons sèches (décembre-mars et juillet-septembre) (http://www. mairiedegrandbassam.ci/fr/don néesphysiquesgénérales). Selon sa morphologie, cette lagune présente des caractéristiques identiques à celle de la lagune estuarienne définie par une barrière courte, des courants de marées et d'apports fluviatiles (Maanan, 2007). de la dégradation des écosystèmes côtiers (Turki, 2004). Le principal objectif de notre étude était de contribuer à la connaissance des Chrysophyta des eaux lagunaires, tout particulièrement par la description succincte et la mise en évidence de l'écologie de quelques taxons rencontrés dans la lagune Vodroboué et son embouchure.

En effet, cette lagune communique avec le fleuve Comoé par l'intermédiaire d'une courte barrière formant ainsi une embouchure et peut être classée selon sa morphologie dans la catégorie de type 2 en se référant à la classification de Lankford (1977). Les berges de cette lagune sont occupées par une invasion de macrophytes flottants et ses eaux présentent des caractéristiques d'eau douce suite à l'échange permanent de celles-ci avec le fleuve Comoé (Kouamé et al., 2009 ; Kouadio et al., 2017). La station E (427000 UTM latitude nord et 589000 UTM longitude ouest) est située à l'embouchure fleuve Comoé-lagune Vodroboué, tandis que les stations V1 (431800 UTM latitude nord et 578200 UTM longitude ouest), V2 (433400 UTM latitude nord et 579800 UTM longitude ouest) et V3 (436000 UTM latitude nord et 579000 UTM longitude ouest) sont localisées dans le plan d'eau lagunaire. 


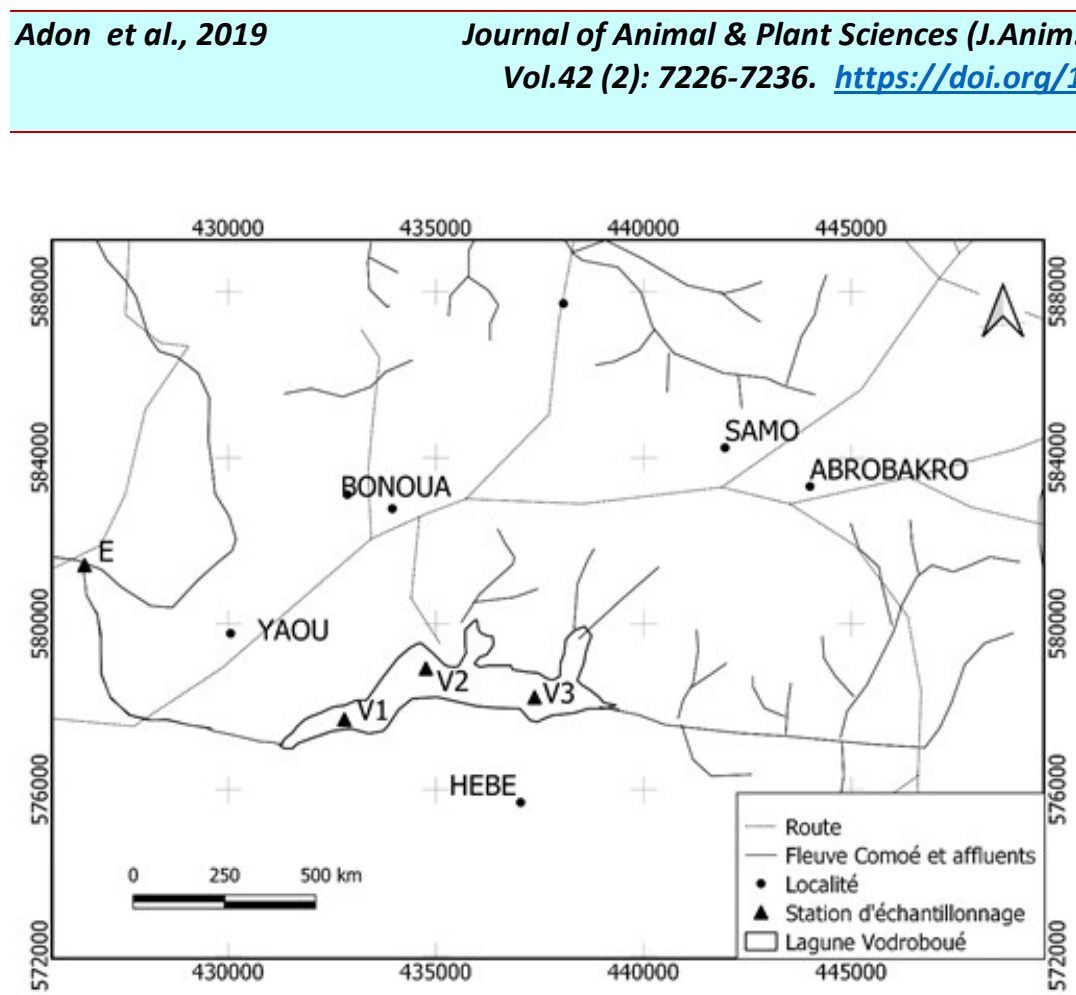

Figure 1 : Carte de localisation des stations d'échantillonnages

3.2 Mesure des paramètres physicochimiques: Les mesures des paramètres physico-chimiques tels que le $\mathrm{pH}$, la température, de conductivité, et les prélèvements d'échantillons dans le but de doser les nutriments (nitrates et phosphates) ont été réalisées entre 8 et $12 \mathrm{~h}$ dans les quatre stations d'échantillonnage pendant les périodes 2014-2015 et 2016-2017. Pour ce qui concerne le $\mathrm{pH}$, la température et la conductivité, les mesures ont été effectuées in situ à l'aide du multimètre portable de marque HANNA. Les concentrations de nitrates et de phosphates ont été respectivement dosées selon la technique auto-analyse II (Tréguer et Le Corre, 1975) avec une précision estimée à $0,01 \mu \mathrm{mol} \mathrm{L^{-1 }}$ et la méthode standard colorimétrique de Grasshoff et al. (1983) avec une précision estimée à $\pm 0,01$ $\mu \mathrm{mol} \mathrm{L}{ }^{-1}$.

3.3 Echantillonnage et analyse des microalgues : Les communautés de microalgues ont été récoltées selon deux modes d'échantillonnage aux mêmes périodes de mesure des paramètres physico-chimiques. Le premier mode d'échantillonnage a consisté au prélèvement des organismes à l'aide de la bouteille de Niskin et du filet à plancton de 20 $\mu \mathrm{m}$ de mailles pour ce qui est des communautés pélagiques, tandis que le second mode a concerné la récolte des communautés épiphytiques sur les systèmes racinaires des macrophytes végétaux tels que Eichhornia crassipes, Echinochloa pyramidalis, Centrosema pubescens, Centrosema sp., Commelina bengalensis, Commelina sp., Cuscuta sp., Cyclosorus striatus, Cyclosorus sp., Cyperus sp., Cyrtosperma senegalense, Lemna paucicostata, Ludwigia sp., Nymphea lotus, Panicum rupens, Panicum sp., Paspalum vaginatum, Paspalum sp., Penicetum purpureum, Pistia stratiotes, Salvinia molesta et Scleria sp. rencontrés aux stations d'echantillonnage suivant un quadrat de $0,5 \mathrm{~m}^{2}$. Les échantillons prélevés ont été fixés au Lugol avec ajout de quelques gouttes de formol puis conservés dans des piluliers. Les observations et les prises de vues des taxons ont été réalisées à l'aide du Microscope Électronique à Balayage-Spectrométrie à Dispersion d'Energie (MEB-SDE) de marque Supra 40 VP Zeiss du laboratoire du Centre d'Analyses et de Recherche, PETROCI Holding, Abidjan, Côte d'Ivoire. Pour ce faire, la préparation des microalgues pour leur observation a été effectuée selon la méthodologie préconisée par Couté (2002), qui consiste en leur isolement à la micropipette. Ces microorganismes ont été ensuite lavés à l'eau 
distillée (pour éliminer les débris abondants dans les récoltes) et déshydratés par la technique du point critique avec comme milieu final le dioxyde de carbone liquide. Montées sur les portes-objets couverts d'un disque adhésif double-face préalablement carboné, les taxons ont été métallisés à l'or-palladium.

\section{RESULTATS}

4.1 Diagnose des taxons: La présente étude ne comporte que des images d'une espèce de Torodinium et des écailles de certains taxons du genre Paraphysomonas et Mallomonas ainsi que de quelques cellules de Mallomonas observés au microscope électronique à balayage (MEB). Les données des paramètres physico-chimiques mesurés dans le plan d'eau lagunaire ont également été utilisées pour les berges du fait des caractéristiques morphologiques de la lagune et du mouvement des fortes marées observées sur tout le plan lagunaire ainsi qu'aux berges. Les taxons observés pour la première fois en Côte d'Ivoire ont été désignés par un astérix.
L'identification des taxons observés a été effectuée à l'aide de clés d'identification et la documentation des auteurs comme Wood (1954), Reid (1972), Gárate-Lizárraga et Muciño-Márquez (2013), Jo et al. (2013) ; Scoble et Cavalier-Smith (2014).

Dinophyta - Dinophyceae - Gymnodiniales Brachidiniaceae

Genre Torodinium Kofoid \& Swezy

Torodinium sp.* (Figure 1) Corps allongé, de 9,12 $\mathrm{x} 3,53 \mu \mathrm{m}$ de dimensions, avec un très petit hypocône réduit à une structure conique. Longueur de cellule 2,58 fois plus longue que la largeur. Le sulcus a une boucle apicale terminale non inversée et la ceinture forme une spirale par la gauche. Stations de prélèvement : espèce récoltée dans le phytoplancton à la station $\mathrm{E}$ et à toutes les stations respectivement durant les périodes 2014-2015 et 2016-2017, et sur les macrophytes dans les stations V1 et V3 durant la période 2016-2017. 

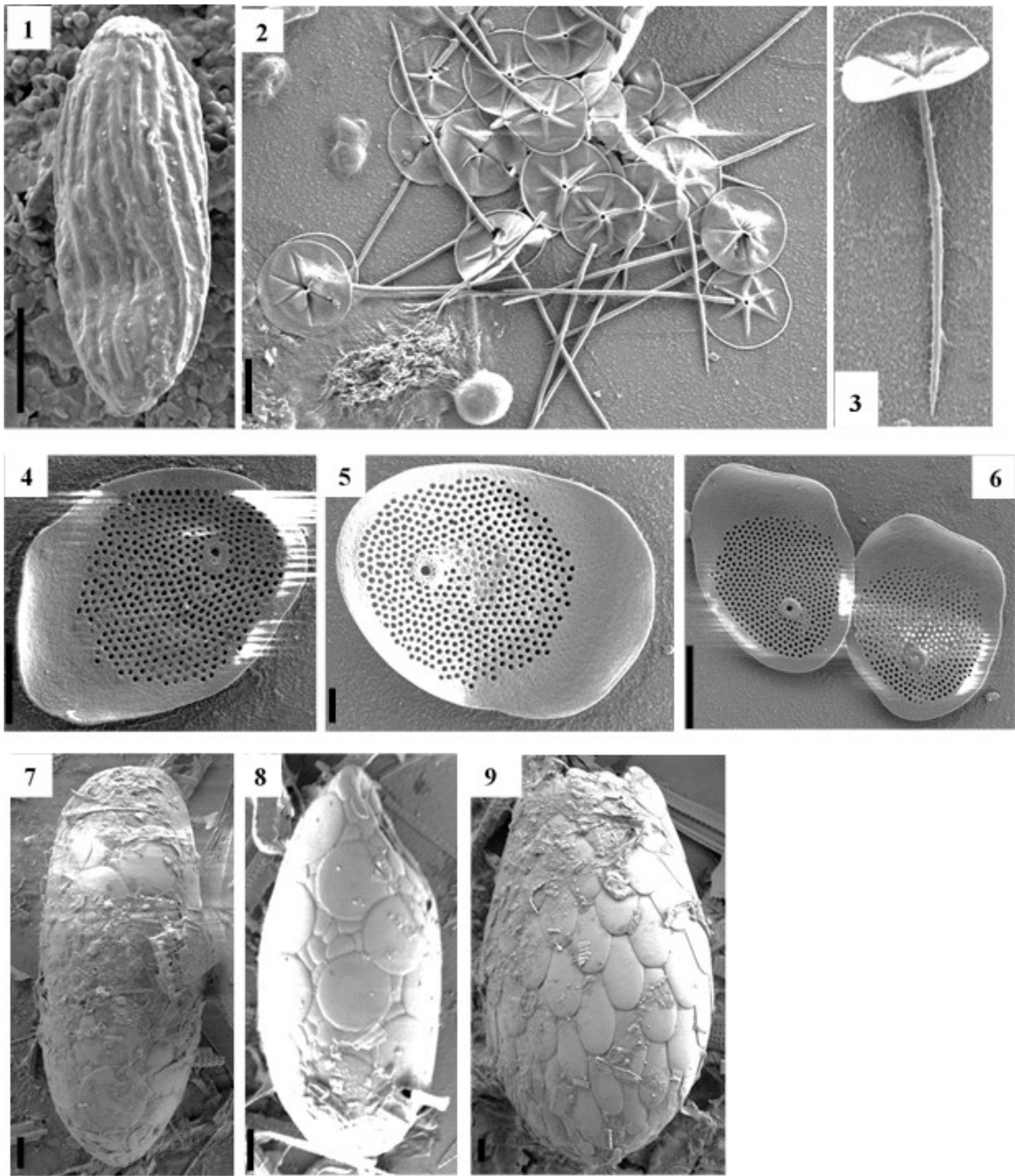

Figures 2-9: Taxons décris, 1 - Torodinium sp. ; 2 à 3- Paraphysomonas uniformis hemiradia sp. n. Type ; 4 à 6-Mallomonas sorohexareticulata ; 7 et 8-Mallomonas sp. ; 9- Mallomonas sp. 1. Le trait à côté des figures représente $1 \mu \mathrm{m}$. 
Chrysophyta - Chrysophyceae - Chromulinales Paraphysomonadaceae

Genre Paraphysomonas De Saedeleer

Selon Compère (1974), les cellules de Paraphysomonas sont solitaires, libres ou fixées, dépourvues de plaste ; membrane couverte d'écailles siliceuses circulaires portant au centre une soie perpendiculaire au plan de l'écaille; deux flagelles inégaux.

Paraphysomonas uniformis hemiradia sp. n. Type* (Figures 2 et 3)

Ecailles siliceuses circulaires de 2,06-2,42 $\mu \mathrm{m}$ de diamètre, portant, au centre une soie de 4,015,98 $\mu \mathrm{m}$ de long. La base de la soie s'évase sur l'écaille avec un nombre de plis radiaux proéminents compris entre 5 et 6 . La longueur du plis radial est comprise entre et 0,65 et 0,87 $\mu \mathrm{m}$. Cette espèce se distingue du type Paraphysomonas uniformis uniformis par le nombre de plis radiaux proéminents.

Stations et périodes de prélèvement: espèce récoltée à toutes les stations $\mathrm{E}, \mathrm{V} 1, \mathrm{~V} 2$ et V3 dans la zone pélagique et sur les macrophytes durant les périodes 2014-2015 et 2016-2017.

Chrysophyta - Synurophyceae - Synurales Mallomonadaceae

Genus Mallomonas Perty

Pour Compère (1974), les cellules de Mallomonas sont flagellées, libres, solitaires. Les cellules sont caractérisées par deux plastes pariétaux, une membrane couverte d'écailles imbriquées parfois pourvues de soies \pm longues, un seul flagelle visible, l'autre fortement réduit.

Mallomonas sorohexareticulata Jo, Shin, Kim, Siver \& Andersen sp. nov.* (Figures 4 ; 5 et 6) : Écailles ovales ou obovoïdes, de dimensions 5,71 - 7,39 x 4,17 - 5,91 $\mu \mathrm{m}$; un bord postérieur entoure environ la moitié du périmètre de l'échelle; grands pores de la plaque de base, régulièrement espacés; région d'échelle antérieure marquée de petites papilles; région à l'échelle postérieure avec un seul grand pore entouré d'une zone hyaline.

Mallomonas sp.* (Figures 7 et 8)
Cellule ovoïde avec un petit pore dépourvu de rebord de dimension 19,34 et 32,26 x 9,10 et $13,40 \mu \mathrm{m}$. Le corps de la cellule est constitué de nombreuses écailles siliceuses de dimensions différentes. Les écailles circulaires plus grandes de 3,85 - 5,6 $\mu \mathrm{m}$ de diamètres, en nombre réduit, recouvrent les plus petites qui couvrent tout le corps de la cellule dont les dimensions sont comprises entre 1,2 - 1,72 x 0,34-0,83 $\mu \mathrm{m}$

Mallomonas sp. 1* (Figure 9)

Cellule ovoïde à ellipsoïde de 25,07 x 14,96 $\mu \mathrm{m}$ de dimension, prolongée dans sa partie antérieure et formée principalement par un col. Ecailles dissemblables ellipsoïdes imbriquées, avec absence de soies, mesurant 3,73 - 4,74 x $1,57-2,59 \mu \mathrm{m}$.

Stations et période de prélèvement : les taxons du genre Mallomonas ont été récoltés dans les stations V1 et V3 du plan lagunaire et sur les macrophytes localisés dans le plan lagunaire à la station embouchure (E) et les berges des stations V2 et V3 durant la période 2014-2015.

Physico-chimie : les taxons du genre Mallomonas et Paraphysomonas uniformis hemiradia ont été observés pour la période 2014-2015 à des températures variant entre 26,3 et $29,74{ }^{\circ} \mathrm{C}(\mathrm{E})$; 27,33 et $30{ }^{\circ} \mathrm{C}(\mathrm{V} 1) ; 27,77$ et $30,2{ }^{\circ} \mathrm{C}$ (V2) et entre 27,37 et $30,4{ }^{\circ} \mathrm{C}(\mathrm{V} 3)$, des valeurs de $\mathrm{pH}$ comprises entre 5,68 et $6,75(\mathrm{E}) ; 6,47$ et 7,69 (V1) ; 6,57 et 7,66 (V2) et entre 6,20 et 7,50 (V3). Les valeurs de conductivité ont varié entre 30 et $37,1 \mu \mathrm{S} / \mathrm{cm}(\mathrm{E}), 19$ et $36,4 \mu \mathrm{S} / \mathrm{cm}$ (V1), 20 et $28,1 \mu \mathrm{S} / \mathrm{cm}$ (V2) et entre 22 et 27,2 $\mu \mathrm{S} / \mathrm{cm}$ (V3). Les nutriments ont été caractérisés par des teneurs de phosphates oscillant entre 0,05 et $0,097 \mathrm{mg} \cdot \mathrm{L}^{-1}(\mathrm{E}) ; 0,015$ et $0,03 \mathrm{mg} \cdot \mathrm{L}^{-1}$ (V1) ; 0,016 et 0,019 mg.L.' (V2) et entre 0,017 et $0,02 \mathrm{mg} . \mathrm{L}^{-1}(\mathrm{~V} 3)$ et de nitrates comprises entre 0,22 et 1,52 mg.L $\mathrm{L}^{-1}(\mathrm{E}), 0,71$ et $0,86 \mathrm{mg} . \mathrm{L}^{-1}$ (V1), 0,56 et $0,96 \mathrm{mg} \cdot \mathrm{L}^{-1}$ (V2) et entre 0,69 et 1,23 mg.L $\mathrm{L}^{-1}$ (V3). Par contre, l'espèce Paraphysomonas uniformis hemiradia a été récoltée durant la période 2016-2017 dans la lagune et son embouchure caractérisés par des valeurs de températures comprises entre 25,9 et $31,9^{\circ} \mathrm{C}$ (E) ; 27 et $32{ }^{\circ} \mathrm{C}(\mathrm{V} 1) ; 27,2$ et $31,6{ }^{\circ} \mathrm{C}$ (V2) et 
entre 27,2 et $31,3{ }^{\circ} \mathrm{C}(\mathrm{V} 3)$; des valeurs de $\mathrm{pH}$ comprises entre 5,19 et 7,5 (E) ; 6 et 6,9 (V1) ; 5,3 et 7 (V2) et 6 et 8,01 (V3). Les valeurs de conductivité ont été comprises entre 14 et 32,3 $\mu \mathrm{S} / \mathrm{cm}(\mathrm{E}) ; 20$ et $25,3 \mu \mathrm{S} / \mathrm{cm}(\mathrm{V} 1) ; 21$ et 25,4 $\mu \mathrm{S} / \mathrm{cm}(\mathrm{V} 2)$ et entre 21 et $26,2 \mu \mathrm{S} / \mathrm{cm}$ (V3), tandis que les teneurs en phosphates ont oscillé entre 0,039 et $0,13 \mathrm{mg} \cdot \mathrm{L}^{-1}$ (E) ; 0,017 et 0,22

\section{DISCUSSION}

La diagnose des taxons a mis en exergue la similarité d'ultrastructure des écailles de Mallomonas sorohexareticulata sp. nov. d'une part, et d'autre part, celle des taxons de Mallomonas sp. observés pour la première fois en Côte d'Ivoire. Cette similarité serait liée à la tolérance des taxons du genre Mallomonas à la variation de la température. La tolérance à la température étant comprise entre 1,5 et $25,5{ }^{\circ} \mathrm{C}$ (Siver, 1991). Les taxons identifiés ont été récoltés à des températures variant entre 26,3 et $29,74{ }^{\circ} \mathrm{C}$ à la station embouchure $(\mathrm{E}) ; 27,33$ et $30^{\circ} \mathrm{C}$ à la station $\mathrm{V} 1 ; 27,77$ et $30,2{ }^{\circ} \mathrm{C}$ dans la station V2 et entre 27,37 et $30,4{ }^{\circ} \mathrm{C}$ pour la station V3. Selon la règle température-taille (Angiletta et Dunham, 2003), des températures plus élevées entraînent une croissance cellulaire plus rapide, puis une taille plus petite des cellules en division. De plus, des températures plus élevées affectent négativement la biogenèse des écailles, interférant avec les processus cellulaires qui produisent les écailles (Pichrtová et Němcová, 2011). En outre, selon certains auteurs (Atkinson et Sibly, 1997 ; Partridge et Coyne, 1997 ; Kindleman et al., 2001 ; Angiletta et al., 2004 ; Yom-Tov et Geffen, 2006), la plasticité température-dépendance a été observée au niveau de la taille du corps des bactéries, des protistes, des plantes et des animaux. Cet état de fait a été observé au niveau des deux cellules de Mallomonas sp. qui ont présentées une variation de taille de la cellule et des écailles. Par ailleurs, au niveau des écailles des espèces de Mallomonas sorohexareticulata, une différence au niveau de la région d'échelle antérieure a été observée. En effet, l'écaille du taxon de la figure 3 n'est pas marquée de petites papilles à la différence des deux autres écailles des taxons mg. $\mathrm{L}^{-1}(\mathrm{~V} 1)$; 0,019 et 0,31 mg.. $\mathrm{L}^{-1}$ (V2) et entre 0,018 et $0,18 \mathrm{mg} \cdot \mathrm{L}^{-1}(\mathrm{~V} 3)$ et celles des nitrates ont varié entre 0,2 et $2,22 \mathrm{mg} \cdot \mathrm{L}^{-1}(\mathrm{E}) ; 0,1$ et 2,66 mg. $\mathrm{L}^{-1}$ (V1) ; 0,2 et 2,66 mg.. ${ }^{-1}$ (V2) et entre 0,1 et $1,77 \mathrm{mg} \cdot \mathrm{L}^{-1}$ (V3). Pour ce qui concerne Torodinium sp., ce taxon a été récolté dans la même masse d'eau que les taxons suscités.

des figures 4 et 5 qui sont caractérisées par la présence de petites papilles au niveau de leurs régions d'échelles antérieures. En outre, la forme des écailles se présente sous la forme obovoïde. Selon (Angiletta et Dunham, 2003), dans l'embranchement des Chrysophyta, les échelles inorganiques produites par les organismes du genre Mallomonas diminuent également de taille en réponse à l'augmentation de la température, de la même manière que les cellules elles-mêmes diminuent. La forme des écailles est devenue moins ronde et plus allongée ou ovale. Ces résultats sont en conformité avec ceux observés par Jo et al. (2013) qui, dans leurs travaux, ont mis en évidence différentes ultrastructures de ces écailles. Concernant le taxon Torodinium sp., sa diagnose a mis en évidence la présence de taxons de petite taille par rapport à ceux observés par Gárate-Lizárraga et al. (2013) dans le golf de la Californie. Ce résultat serait lié à l'influence de la température sur la croissance de cette espèce récoltée à des températures saisonnières variant entre 26,3 et $29,74^{\circ} \mathrm{C}$ pour la période $2014-2015$ et entre 25,9 et $31,9{ }^{\circ} \mathrm{C}$ durant la période 2016-2017 à la station E, entre 27 et $32^{\circ} \mathrm{C}$ à la station V1 et entre 27,2 et $31,3^{\circ} \mathrm{C}$ à la station V3 durant la période 20162017 dans la lagune Vodroboué et son embouchure. Ce taxon se distingue des espèces Torodinium robustum et Torodinium teredo par sa morphologie. En effet, Torodinium robustum est caractérisée par la longueur de la cellule 3,5 fois plus longue que le diamètre, le sulcus a une boucle apicale terminale inversée et la ceinture forme une spirale par la gauche, tandis que Torodinium teredo est caractérisée par la longueur de la cellule 4 fois plus longue que le diamètre, 
une absence de boucle terminale au niveau du sulcus et la largeur de la taille (Gárate-Lizárraga et al., 2013). Selon leur écologie, les taxons de Torodinium robustum, Paraphysomonas uniformis hemiradia sp. n. Type, de Mallomonas sorohexareticulata, et des deux taxons de Mallomonas identifiés au niveau générique appartenant à l'embranchement des Chrysophyta ont été tous récoltés dans la lagune Vodroboué et son embouchure situés dans la zone estuarienne de Grand-Bassam. Les eaux de cette lagune et de son embouchure sont acido-alcalines (Adon et al., 2018) avec de faibles valeurs de conductivité, des concentrations modérées de phosphates $(0,016$ et $0,097 \mathrm{mg} \cdot \mathrm{L}^{-1}$, et, 0,017 et $0,311 \mathrm{mg} \cdot \mathrm{L}^{-1}$ respectivement pour les périodes 2014-2015 et 2016-2017) et de nitrates $\left(0,22\right.$ et $0,097 \mathrm{mg} . \mathrm{L}^{-1}$, et, 0,1 et $2,66 \mathrm{mg} . \mathrm{L}^{-1}$ respectivement pour les périodes 2014-2015 et 2016-2017) durant les périodes d'étude. Pour Reynolds (2006), dans les écosystèmes naturels, la disponibilité réduite de silicone réactive dissoute pour les Chrysophyta peut se produire dans des environnements présentant des valeurs de $\mathrm{pH}$ supérieures à 9. En outre, les chrysophycées

\section{REMERCIEMENTS}

Cette recherche inscrite dans le cadre du projet intitulé "Approche écosystémique de la pêche comme moyen d'exploitation durable des ressources halieutiques dans la zone humide de Grand-Bassam »est financée par le PASRES (Abidjan, Côte d'Ivoire). Nos sincères remerciements sont adressés à cette institution, à toute l'équipe du Pôle Pêche et Aquaculture (PPA) et au Laboratoire d'Environnement et de Biologie Aquatique (LEBA) de l'Université sont typiquement associées à des eaux stagnantes ou des rivières à faible courant, présentant des taux en nutriments faibles à modérés, une alcalinité et une conductivité faibles et un $\mathrm{pH}$ acide à neutre (John et al., 2002, Wehr et Sheath, 2003). En outre, selon De Reviers (2003), les Chrysophyta, microorganismes en majorité dulçaquicoles libres ou fixés ont beaucoup de leurs espèces qui sont typiques des milieux oligotrophes pauvres en phosphore, cependant, certaines vivent dans les milieux eutrophes très riches en matières organiques. Or, les taxons du genre Paraphysomonas et Mallomonas ont été récoltés dans la lagune Vodroboué et son embouchure dont l'hydrologie est caractérisée par des courants de marées et d'apports fluviatiles présentant des caractéristiques physicochimiques similaires à ceux obtenues par John et al. (2002) et Wehr et Sheath (2003) dans leurs travaux. Ces Chrysophyta peuvent être rencontrés dans des écosystèmes d'eau douce, saumâtre et marine (Reviers, 2003 ; Kristiansen, 2005 ; Jo et al., 2013 ; Scoble et Cavalier-Smith, 2014 ; Siver, 2015; Nemcova et al., 2016).

Nangui Abrogoua, aux membres de l'équipe Biologie végétale de l'Université Jean Lorougnon Guédé dans la validation de certains taxons de macrophytes, sans oublier DJINA Agbé Emmanuel et SIDIBE Aïcha, étudiants en Master II de Biodiversité et Gestion Durable des Écosystèmes de ladite Université pour leur contribution dans le prélèvement des échantillons lors des missions d'étude.

\section{REFERENCES BIBLIOGRAPHIQUES}

Adon MP, Niamien-Ebrottié JE, Kouassi BAT, Ouattara A et Gourene G: 2018. Diatomées des eaux de l'embouchure fleuve Comoé-lagune Vodroboué et de la lagune Vodroboué au Sud-Est de la Côte d'Ivoire (sud-est, Côte d'Ivoire). Rev.Ramres 6 (1): 30 - 41.
Angiletta, MJ et Dunham AE: 2003. The temperature-size rule in ectotherms: Simple evolutionary explanations may not be general. The American Naturalist 162 (3): $332-342$.

Angiletta MJ, Steury TD et Sears MW: 2004: Temperature, growth rate, and body size in ectotherms: Fitting pieces of a 
life-history puzzle. Integrative and Comparative Biology 44: 498 - 509.

Atkinson D et Sibly RM: 1997. Why are organisms usually bigger in colder environments? Making sense of a life history puzzle. Trends in Ecology and Evolution 12: 235 - 239.

Brennan L et Owende P: 2010. Biofuels from microalgae-A review of technologies for production, processing, and extractions of biofuels and co-products. Renewable and Sustainable Energy Reviews 14: 557 $-577$.

Coute A: 2002: Biologie et microscopie électronique à balayage. Mémoires de la SEF 6: $31-44$.

Da KP 2007: Étude taxinomique du phytoplancton dulçaquicole des masses d'eau lentiques et lotiques de quelques sites au Sud de la Côte d'Ivoire, entre les fleuves Bandama et Bia : apport de la microscopie électronique à balayage. Thèse d'État. Université de CocodyAbidjan (Côte d'Ivoire). 401 pp.

De Reviers B: 2003. Biologie et phylogénie des algues. Belin, Paris. Collection Sup Sciences. Tome 2. 255 pp.

FDR: 2005. Fiche descriptive sur les zones humides Ramsar. Grand Bassam, Côted'Ivoire. 17 pp.

Gárate-Lizárraga I et Muciño-Márquez RE: 2013. New data on the distribution of Torodinium robustum and $\mathrm{T}$. teredo (Dinophyceae: Gymnodiniales) in the Gulf of California. Check List 9 (4): 809 $-812$.

Grasshoff K, Ehrhardt M et Krelling K: 1983. Methods of Seawater Analysis. Verlag Chemie Weinheim, pp 143-187.

Jo BY, Shin W, Kim HS, Siver PA et Andersen RA: 2013. Phylogeny of the genus Mallomonas (Synurophyceae) and descriptions of five new species on the basis of morphological evidence. Phycologia 52 (3): $266-278$.

John DM, Whitton BA et Brook AJ: 2002. The Freshwater Algal Flora of the British Isles. Cambridge University press, The
Natural History Museum, (London, England). 702 pp.

Kindleman P, Dixon AFG et Dostálková I: 2001. Role of ageing and temperature in shaping reaction norms and fecundity functions in insects. J. of Evolutionary Biology 14: $835-840$.

Kofoid CA et Swezy O: 1921. The free-living unarmored Dinoflagellata. City: Memoirs of the University of California, USA. 562 pp.

Konan ES: 2014. Distribution spatiotemporelle du peuplement phytoplanctonique en relation avec les facteurs abiotiques de la lagune de fresco (côte d'ivoire). Thèse de doctorat de l'Université Félix HOUPHOUËTBOIGNY, Abidjan, Côte d'Ivoire. 197 pp.

Kouadio JK, Annigbé JE, Konan N et Boua CA: 2017. Length-weight relationship and condition factor for 18 fish species from Ono, Kodjoboué and Hébé lagoons, Southeast of Ivory Coast. International Journal of Fisheries and Aquatic Studies 5 (6): $13-18$.

Kouame MLO, Egnankou MW et Traore D: 2009. Ordination et classification de la végétation des zones humides du sudest de la Côte d'Ivoire. Agronomie Africaine 21(1): 1-13.

Kristiansen J: 2005. Golden algae: A biology of chrysophytes. Koeltz Scientific Books, Konigstein, Germany. 167 pp.

Laë R: 1992. Les pêcheries artisanales lacunaires Ouest- Africaines : échantillonnage et dynamique de la ressource et de l'exploitation. Éditions de I'ORSTOM. Institut français de recherche scientifique pour le développement en coopération. Collection Etudes et Thèses,

Thèse soutenue le 17 décembre 1990 à l'université de Bretagne occidentale, Paris. ISSN: 0767-2888/ISBN: 2-70991098-5.

Lozo N, Berté S, Komoé K, Yao S et Kouamélan P: 2013. Bacillariophyceae 
(Heterokontophyta) from Bandana River in Côte d'Ivoire, West Africa. Journal of Animal and Plant Sciences 20 (2): 3113 - 3121.

Nemcova Y, Pusztai M, Skaloudova M et Neustupa J: 2016. Silica-scaled chrysophytes (Stramenopiles, Ochrophyta) along a salinity gradient: a case study from the Gulf of Bothnia western shore (northern Europe). Hydrobiologia 764 (1): 187 - 197.

Maanan M: 2007. Etude sédimentologique du remplissage de la lagune de sidi moussa (côte atlantique

Marocaine) caractérisations granulométrique, minéralogique et géochimique. Thèse de doctorat de la Faculté des Sciences El Jadida, soumis le 22 Jan 2007, Maroc. HAL, https:/ / tel.archives-ouvertes.fr

Partridge L et Coyne JA: 1997. Bergmann's rule in ectotherms: Is it adaptive? Evolution 51: $632-635$.

Person J (coord.): 2011. Livre Turquoise : Algues, filières du futur, Actes du colloque Algues, Adebiotech, Romainville, $182 \mathrm{pp}$.

Philibert J: 1968. La microscopie électronique à balayage et ses applications métallurgiques. Revue de Physique Appliquée, 3 (4): 325 - 330.

Pichrtová M. et Němcová Y.: 2011. "Effet de la température sur la taille et la forme des écailles de silice chez Synura petersenii et Mallomonas tonsurata (Stramenopiles)". Hydrobiologia 673 (1): 1 - 11.

Reid PC: 1972. Dinoflagellate cyst distribution around the British Isles. Journal of Marine Biological Association United Kingdom 4(52): 939 - 944.

Röch C et Posten C: 2012. (Editors). Introduction to the thematic Focus. Technik folgen abschatzung Theorie und Praxis. Karlsruher institute für Technologie, pp. 80-85.

Reynolds CS: 2006. Ecology of phytoplankton. Cambridge, Cambridge University Press, 552 pp.
Scoble JM et Cavalier-Smith T: 2014. Scale evolution in Paraphysomonadida (Chrysophyceae): sequence phylogeny and revised taxonomy of Paraphysomonas, new genus Clathromonas, and 25 new species. European journal of protistology 50 (5): $551-592$.

Siver PA: 1991. The Biologie of Mallomonas. Developments in Hydrobiology, Volume 63, Pays-Bas. 228 pp.

Siver PA: 2015. The Synurophyceae. Wehr JD, Sheath RG et Kociolek JP (Editors.), Freshwater algae of North America: Ecology and classification, 2nd Edition, Academic Press, San Diego, California, USA. $650 \mathrm{pp}$.

Spolaore P, Joannis-Cassan C, Duran E et Isambert A: 2006. Commercial applications of microalgae. Journal of Bioscience and Bioengineering 101: 87 $-96$.

Tréguer P et Le Corre P: 1975. Manuel d'analyses des sels nutritifs dans l'eau de mer. Utilisation de l'auto-analyzer II Technicon, 2nd Edition. France, Brest, Université de Bretagne Occidentale. 1 p.

Turki S: 2004. Suivi des microalgues planctoniques toxiques dans les zones de production, d'elevage des mollusques bivalves et d'exploitation des oursins du nord de la tunisie. Bulletin de l'Institut National des Sciences et Technologies de la Mer de Salammbô 31: 83 - 96.

Wehr JD et Sheath RG: 2003. Freshwater Algae of North America: Ecology and Classification. Academic Press, San Diego. 918 pp.

Wood EJF: 1954. Dinoflagellates in the Australian region. Australian Journal of Marine and Freshwater Research 5 (2): $171-352$.

Yom-Tov Y et Geffen E: 2006. Geographic variation in body size: the effects of ambient temperature and precipitation. Oecologia, 148: 213 - 218. 